21st Particles and Nuclei International Conference (PANIC 2017)

International Journal of Modern Physics: Conference Series

Vol. 46 (2018) 1860086 (5 pages)

(C) The Author(s)

DOI: $10.1142 / \mathrm{S} 2010194518600868$

\title{
Detector Performance and Physics Potential at CEPC
}

\author{
Hao Liang* ${ }^{*} \dagger$ and Manqi Ruan*, \\ Experimental Physics Division, Institute of High Energy Physics, \\ Beijing 100049, China \\ †liangh@ihep.ac.cn \\ ‡manqi.ruan@ihep.ac.cn
}

Published 3 May 2018

\begin{abstract}
The Circular Electron Position Collider (CEPC) is a future $Z$ and Higgs bosons factory, which targets precision electroweak and Higgs measurements. The simulation, reconstruction, analysis chain has been established at CEPC. The detector design parameters were optimized according to several performances of key physical objects. The physics potential has been studied for the detector geometry CEPC_v1.
\end{abstract}

Keywords: CEPC; Higgs Factory; PFA.

\section{Introduction}

The Higgs boson was discovered at the LHC in 2012. Precision measurements of the Higgs boson properties become a focus of the experimental High Energy Physics. The precision Higgs and electroweak measurements allow us to access new physics indirectly and shed light on unanswered fundamental questions, e.g hierarchy problem. The proposed the Circular Electron Position Collider (CEPC) as a $Z$ and Higgs boson factory, can meet this requirement. CEPC has a circumference of 100 kilometers. After the operations of CEPC, a proton-proton collider (SPPC) can be constructed in the same tunnel. The SPPC can reach unprecedented high energy to search new physics

CEPC could be operated at center of mass energy of about $91 \mathrm{GeV}$ as a $Z$ factory. About $10^{10} Z$ bosons will be produced for precision eletroweak measurements. CEPC could be also operated at center of mass energy of $250 \mathrm{GeV}$. About $10^{6}$ Higgs bosons and $10^{8} \mathrm{~W}$ bosons will be produced. If everything goes well, the data taking will start in 10 years (in 2027).

\footnotetext{
*On the Behalf of CEPC Collaboration.
}

This is an Open Access article published by World Scientific Publishing Company. It is distributed under the terms of the Creative Commons Attribution 4.0 (CC-BY) License. Further distribution of this work is permitted, provided the original work is properly cited. 


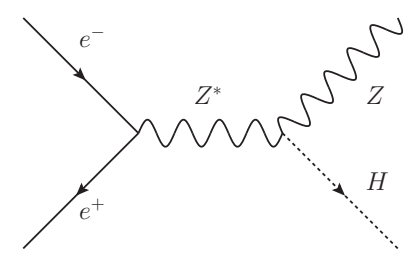

Fig. 1. Feynaman diagram of Higgs-straghlung process.

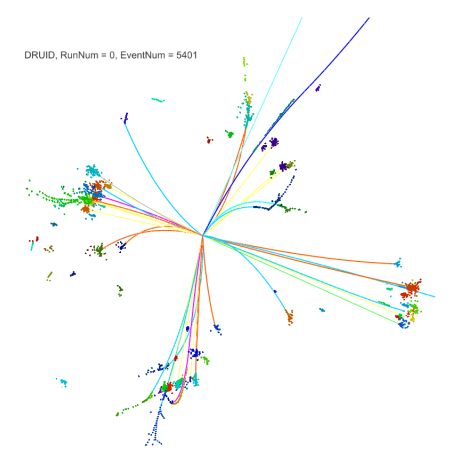

Fig. 2. Display of a four jets event at CEPC.

LHC has strong ability to find new physics, but a lepton collider like CEPC has advantages in precision measurements. At a lepton collider, the momenta of incident particles are well known. Benefiting from this, the Higgs can be constructed via the recoil $Z$ for Higgs-strahlung process, as shown in Fig. 1. That allows us to study the exotic or invisible decays of Higgs. Also, a lepton collider has a very clean collision environment. This have three advantages. Firstly, the backgrounds cross sections at lepton collider are of orders of magnitude smaller than those at hadron collider. The Higgs yield during the lifetime of LHC can be at level of $10^{7}$, which is one order of magnitude larger than that at CEPC. However, the event selection efficiency at LHC is only about $10^{-3}$ suffering from the large backgrounds. In contrast, the efficiency at CEPC is of order of one. Secondly, the collision events can be triggered one by one at CEPC, thus physics analysis doesn't suffer from the pile-up. See Fig. 2. Finally, the standard model backgrounds can be calculated precisely by theory with well known initial condition, thus can be well understood.

\section{Detector Design}

The CEPC detector consists of a set of sub-detectors. From inside to outside, they are, vertex detector, silicon tracker, main tracker (TPC), ECAL, HCAL, and the magnetic system/muon detector. The geometry of the whole detector is shown in the Fig. 3. It is challenging to design a detector that meets the physics requirements. 


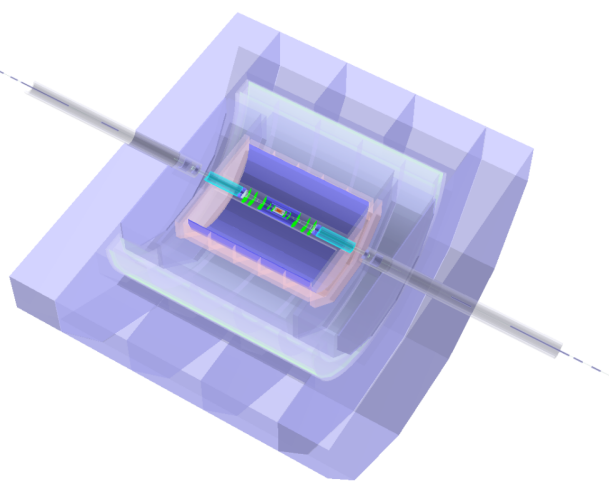

Fig. 3. The geometry of the CEPC detector.

The key idea of CEPC detector is the Particle Flow Algorithm (PFA) oriented concept. With this concept, the ECAL was designed to be with high granularity, so that each particle can be separated from each other. Once we can do the separation, energies and momenta of each physical objects are obtained from the most suited sub-detector. The momenta and energies of charged particles will be obtained from the tracker, the photons from the ECAL, and the neutral hadrons from the HCal. The PFA will greatly improve the momentum and energy performances. A dedicated PFA package, Arbor ${ }^{1}$, has been developed and validated in the full simulation at CEPC.

The parameters of the detector have been optimized based on the full simulation study. In the full simulation, a chain of event generation, geant4 based simulation of interaction of particles with detector, particles reconstruction, and analyses are fully performed. The key design parameters are listed as following:

- Acceptance: $|\cos \theta|<0.99$

- Radius of TPC: $1.8 \mathrm{~m}$

- B field: $3 \mathrm{~T}$

- ToF: $50 \mathrm{ps}$

- ECAL thickness: $84 \mathrm{~mm}$

- ECAL cell size: $10 \mathrm{~mm}$

- HCAL thickness: $1 \mathrm{~m}$

- Number of layers in HCAL: 40

The TPC is optimized according to the study of branching ratio for Higgs boson to di-muon decay. ToF is requested by pi-kaon separation at $Z$ pole. The thickness and cell size of ECal is determined by the study of Higgs to di-photon. The thickness and number of layers of HCAL is optimized according to the Higgs events at 250 $\mathrm{GeV}$. 


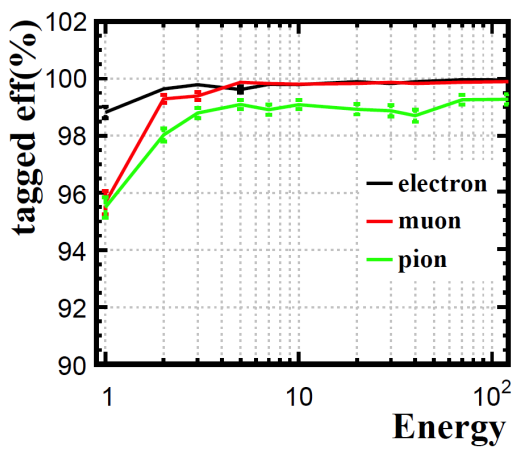

(a) Light lepton ID efficiency $>99.5 \%$

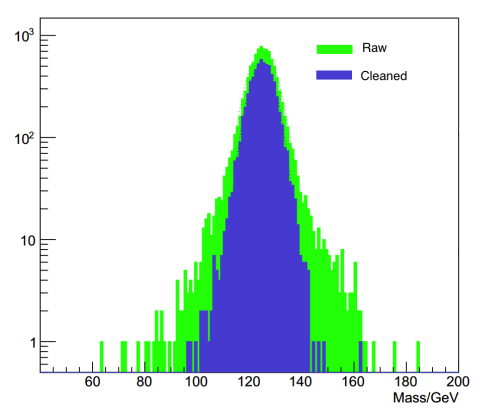

(c) Mass width for $H \rightarrow g g$

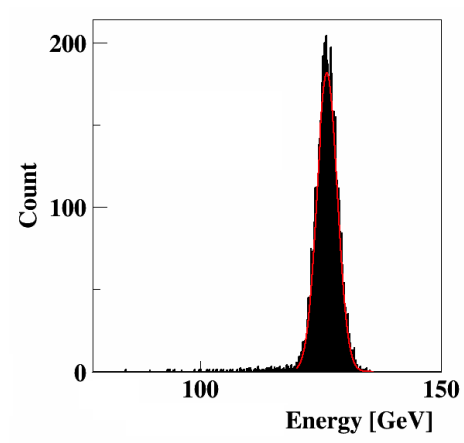

(b) Mass width for $H \rightarrow \gamma \gamma$

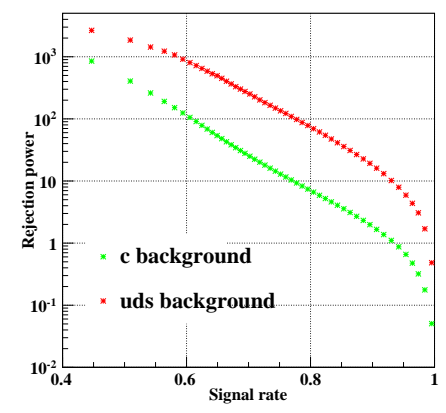

(d) $b$ tagging performance

Fig. 4. Detector key performance.

\section{Detector Performance}

The detector performance of the optimized detector is shown in Fig. 4. (A) In the the lepton identification (ID), information from TPC $\mathrm{d} E / \mathrm{d} x$ and ToF, combined with informaiton from Ecal and HCal, are used. For the charged track with energies larger than $2.0 \mathrm{GeV}$, the efficiencies of leptons are larger than $99.5 \%$, and the pion missed ID rate is at the $1 \%$ level ${ }^{2}$. (B) The invariant mass distribution for $H \rightarrow g g$ reflects the overall detector performance: main tracker, ECAL, HCAL, and PFA ${ }^{3}$. (C) The energy resolution of $H \rightarrow \gamma \gamma$ is referred as the physics benchmark to character ECAL performance. The optimization has been studied for a simplified version of CEPC baseline detector ${ }^{3}$, where the mass resolution of $1.7 \%$ can be achieved, as shown in the Fig. 4(c). For the CEPC baseline detector, mass resolution of $2.2 \%$ can be achieved ${ }^{4}$. (D) The $b$ tagging efficiency represents the performance of the vertex detector. This performance will play significant role in separating Higgs decays to $b \bar{b}, c \bar{c}$, and $g g$. At $b$ tagging efficiency of $80 \%$, the $c$ and light quarks fake rates are about one tenth and one hundredth, respectively. 

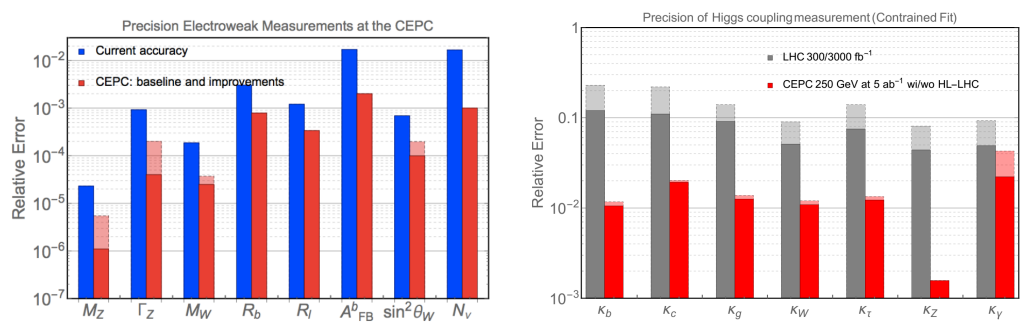

Fig. 5. Left panel: the electroweak measurement precision in $Z$ pole run at CEPC. The current accuracy is obtained from the Particle Data Group. Right panel: the Higgs measurements precision in the Higgs run at CEPC.

In summary, the detector performance has been fully established in simulation, reconstruction, and analysis chain. A dedicated reconstruction algorithm is able to reconstruct every physics objects properly.

\section{Physics Potential}

The physics potential has been studied ${ }^{5}$ with detector geometry CEPC_v1 and magnetic field of 3.5T. See Fig. 5. For almost all electroweak parameters, there are improvements of about one order of magnitude compared to the current best accuracy. Except the coupling of Higgs to $\gamma$, the couplings of Higgs also have near one order of magnitude improvements. All precisions of Higgs couplings are at percentage level. Especially, the Higgs-strahlung cross section, thus the coupling of Higgs to $Z$ boson, can be measured by reconstructing the Higgs via the recoil mass of $Z$ without needs of Higgs decay width or branching ratios. The result of this coupling is most precise, which is at level of $10^{-3}$.

\section{Conclusion}

Numerous progresses have been made with detector optimization, dedicated reconstruction and analysis studies. Accelerator and detector design of CEPC is feasible to achieve the physics goal. CEPC can measure the electroweak and Higgs parameters with unprecedented precision: For both electroweak and Higgs measurements, an order of magnitude of improvements can be made compared to the current best and the future High Luminosity LHC (HL-LHC) project, respectively.

\section{References}

1. M. Ruan, H. Videau, Arbor: a new approach to the particle flow algorithm, in proceedings of Calorimetry for the High Energy Frontier (2013), p. 316, arXiv: 1403.4784.

2. D. Yu, M. Ruan, H. Videau, Eur.Phys.J. C 77, no.9, 591 (2017) arXiv:1701.07542.

3. H. Zhao, M. Ruan, C. Fu, D. Yu, B. Ma, Z. Wang, T. Hu, arXiv:1712.09625.

4. M. Ruan, Simulation, reconstruction and software at the CEPC, presentation at CEPC workshop, April 2016, Beijing.

5. The CEPC-SPPC Study Group, CEPC-SPPC Preliminary Conceptual Design Report, Vol. 3 (2015). 